Bull. Mater. Sci., Vol. 22, No. 2, April 1999, pp. 85-87. (C) Indian Academy of Sciences.

\title{
Recovery characteristics of optical hydrogen sensor using Pd thin film: Behaviour of three-stage hydrogen desorption
}

\author{
YOSHIKI OKUHARA and MASASUKE TAKATA* \\ Department of Electrical Engineering, Nagaoka University of Technology, Kamitomioka 1603-1, Nagaoka, \\ Niigata 940-21, Japan
}

\begin{abstract}
The recovery behaviour of optical hydrogen sensor using Pd nlm has been investigated. The change in reflectance with hydrogen desorption indicated distinctive characteristics with three-stage curve which depended on thickness of the Pd film. Moreover, the distinction became clearer with increasing number of absorption-desorption cycles. These behaviours were related to the change in pressure concentration isotherm.
\end{abstract}

Keywords. Hydrogen sensor; optical sensing; recovery characteristics; palladium.

\section{Introduction}

In recent years, hydrogen gas has been expected to play an important role as a clean energy resource (Christofides and Mandelis 1990). It is therefore essential to develop a sensing device for monitoring hydrogen gas because it is explosive with wide range of concentration and low ignition energy. An optical hydrogen sensor is one of the candidates because of its excellent characteristics at room temperature and high safety (Oh et al 1993). It consists of a thin film of catalytic metal such as palladium (Pd) deposited on glass substrate or optical fibre end. The change in optical properties of Pd with formation of hydride is utilized for the detection. Most devices have lower recovery rate related to dehydrogenation than response rate connected with hydrogenation. Therefore, a clear understanding of the dehydrogenation process is important to realize the high recovery rate.

In the present work, the recovery behaviour of the optical hydrogen sensor using $\mathrm{Pd}$ thin film has been investigated and interpreted in terms of pressure concentration isotherm.

\section{Experimental}

Since blister formation was confirmed in relatively thick Pd film after exposure to hydrogen gas (Hamagami et al 1999), tungsten (W) film as a buffer layer was inserted between Pd film and glass substrate (Corning \#7059) (Okuhara et al 1999). All films were prepared by a radio-frequency magnetron sputtering apparatus (Anelva, SPF-332H) in which $99.99 \%$ pure $\mathrm{Pd}$ and $\mathrm{W}$ plates were equipped. After pumping below $5 \times 10^{-5} \mathrm{~Pa}, \mathrm{Ar}$ gas of $1 \mathrm{~Pa}$ was introduced into the chamber. The thickness of

*Author for correspondence the Pd film was varied from $10 \mathrm{~nm}$ to $100 \mathrm{~nm}$, and that of the W film was $100 \mathrm{~nm}$. Henceforth, the double-layered film is referred to as $P d / W$ film.

For the measurement of optical properties, the specimen was placed in the cell with glass window. The hydrogen partial pressure in the atmosphere was controlled by changing the flow ratio of $\mathrm{H}_{2}$ to Ar. The Pd side of the specimen was irradiated perpendicularly with chopped light from a semiconductor laser (wavelength: $680 \mathrm{~nm}$ ) and the reflected light was detected with a Si-pin photodiode. All measurements were conducted at room temperature and atmospheric pressure. The phases of $\mathrm{Pd}$ and Pd hydride films were estimated by using an X-ray diffractometer (RIGAKU, RINT-1500).

\section{Results and discussion}

Figure 1 shows the change in reflectance of $\mathrm{Pd} / \mathrm{W}$ film with hydrogen absorption and desorption. The initial reflectance was independent of thickness of the Pd film. The total reflectance change increased with increasing thickness of the Pd film up to $75 \mathrm{~nm}$ thick, which was attributed to increase of $\mathrm{H} / \mathrm{Pd}$ ratio in $\mathrm{Pd}$ hydride film (Lee and Glosser 1985). The recovery with hydrogen desorption was obviously slower than the response with hydrogen absorption. And this tendency increased with increasing thickness of the Pd film due to an increase in total amount of absorbed hydrogen. Moreover, distinctive recovery curves with three stages were observed for the Pd film of more than $75 \mathrm{~nm}$ thickness. Accordingly it was confirmed that thicker Pd film exhibited a greater change in reflectance although its recovery rate was lower.

Figure 2 presents the changes in reflectance of $\mathrm{Pd} / \mathrm{W}$ film with repetitional hydrogen absorption and desorption. $P d$ and $W$ were $100 \mathrm{~nm}$ each. With increasing number 
of absorption-desorption cycles, the recovery slowed down and three-stage curve became more distinct.

$\mathrm{X}$-ray diffraction analysis revealed that the $\mathrm{Pd}$ film transformed to $\beta$-phase $\mathrm{Pd}$ hydride after exposure to hydrogen gas. It is known that $\beta$-phase Pd hydride transforms to $\alpha$-phase with decreasing hydrogen content (Lewis 1967). Figure 3 shows change in intensity of $\mathrm{X}$-ray diffraction peak assigned to (111) $\beta$-phase Pd hydride during hydrogen desorption process. The time for disappearance of the $\beta$-phase Pd hydride increased with the number of absorption-desorption cycles. This tendency corresponds to the recovery characteristics shown in figure 2 , which suggests that three-stage curve may be explained by the phase transformation of $\mathrm{Pd}$ hydride thin film.

In order to clarify the above mentioned consideration, the phase boundary was determined. Figure 4 presents the total reflectance change $(\Delta R)$ as a function of hydrogen partial pressure at room temperature. The hysteresis was observed as follows: plateau regions were shown at hydrogen partial pressure of $2.0 \sim 3.0 \mathrm{kPa}$ for absorption and $0.2 \sim 0.3 \mathrm{kPa}$ for desorption. This result is similar to that shown in pressure concentration isotherm (Lee and Glosser 1985), in which the hydrogen pressure at plateau region was about $1.6 \sim 1.7 \mathrm{kPa}$ for absorption and about $0.6 \sim 0.7 \mathrm{kPa}$ for desorption. It can be said that the boundaries between first-stage and second-stage and between second-stage and third-stage in figure 2 coincide with the phase boundaries between $\beta$-phase and

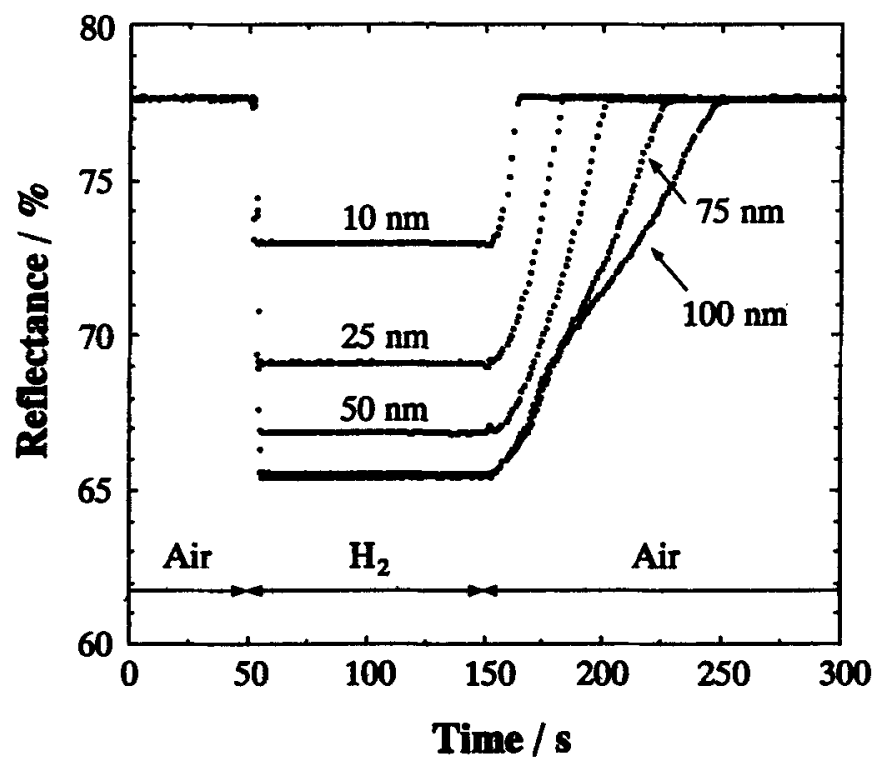

Figure 1. Changes in reflectance of $\mathrm{Pd} / \mathrm{W}$ films with hydrogen absorption and desorption at room temperature. Buffer layer of W (100 nm thick) was inserted between Pd film and glass substrate. The thickness of $\mathrm{Pd}$ film was varied from $10 \mathrm{~nm}$ to $100 \mathrm{~nm}$. plateau region and between plateau region and $\alpha$-phase in figure 4 , respectively.

As shown in figures 1 and 2, three-stage curve became more distinct with increasing thickness of Pd film and number of absorption-desorption cycles. These behaviours may be attributed to the sharpening of pressure concentration isotherm, namely, the slope of the plateau region

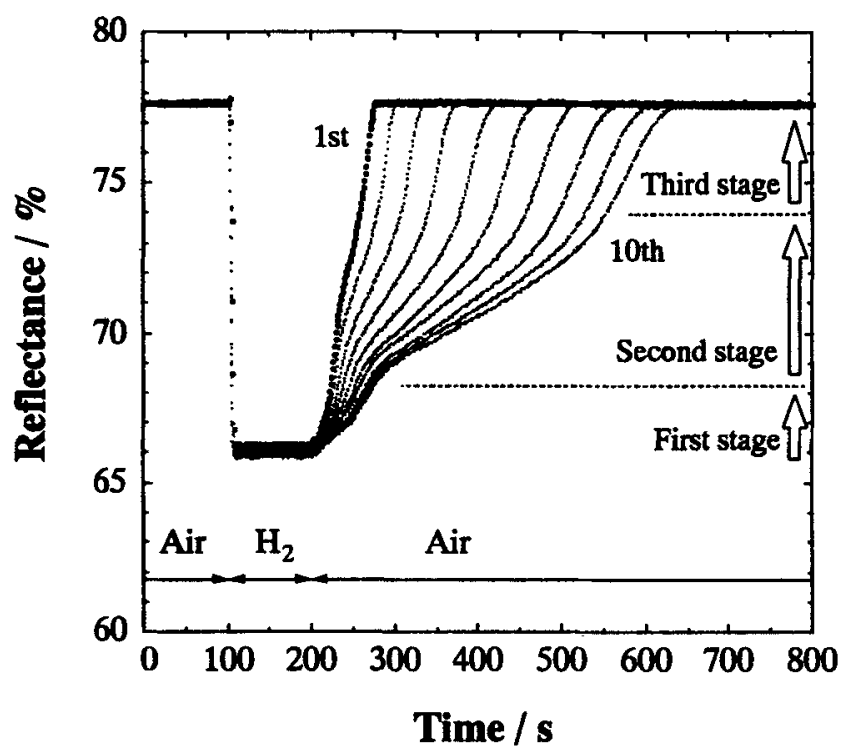

Figure 2. Change in reflectance of $\mathrm{Pd} / \mathrm{W}$ film with hydrogen absorption and desorption at room temperature. $P d$ and $W$ were $100 \mathrm{~nm}$ each. The change in atmosphere (air $\rightarrow \mathrm{H}_{2} \rightarrow$ air) was repeated ten times.

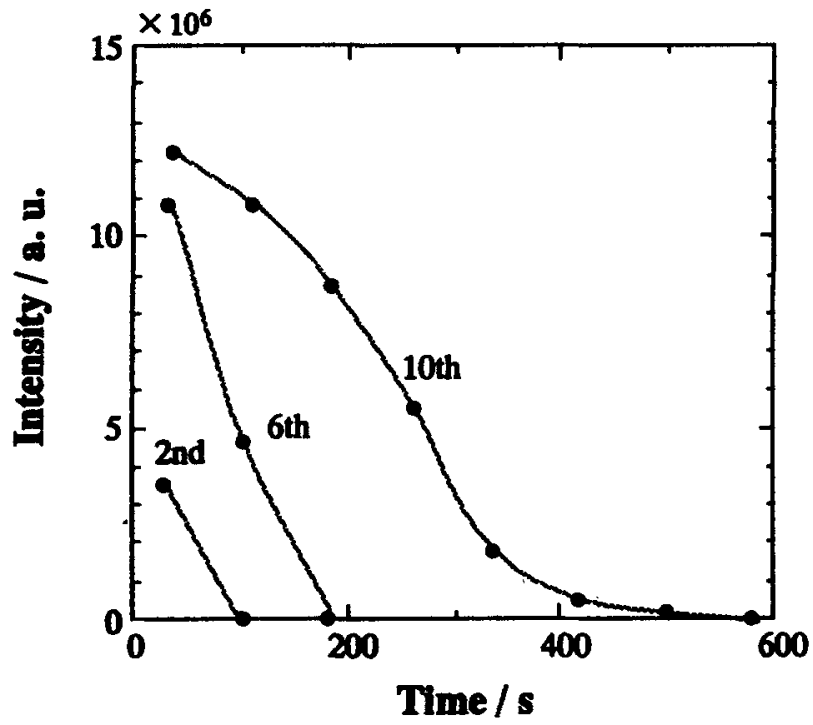

Figure 3. Change in intensity of $\mathrm{X}$-ray diffraction peak assigned to (111) $\mathbf{P d H}_{0 \times \pi \times 6}(\beta$-phase) during hydrogen desorption process. The intensity of the peak was measured immediately after the ambient was replaced from $\mathrm{H}_{2}$ to air. 


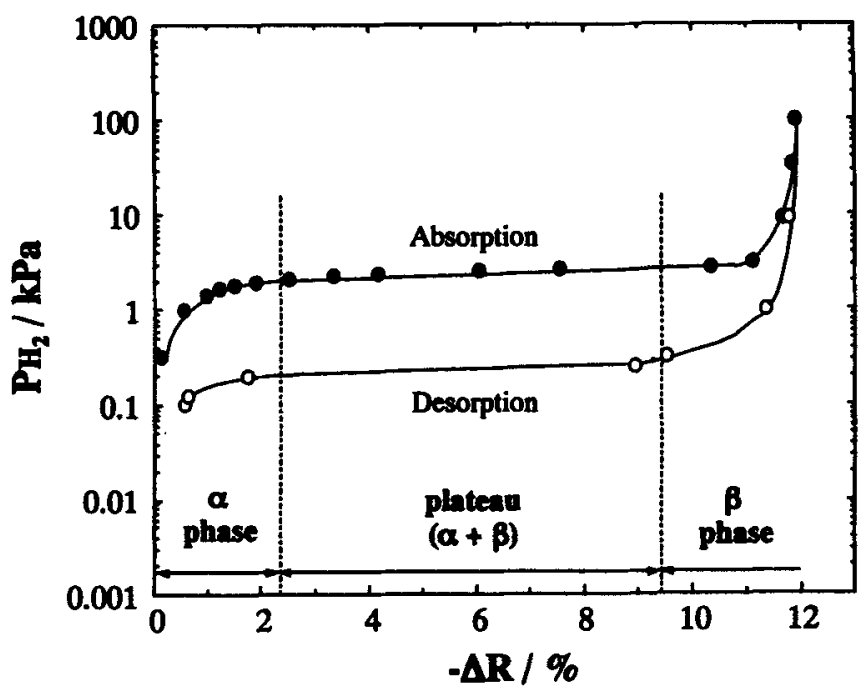

Figure 4. Dependence of $-\Delta R$ (total reflectance change for $\mathrm{Pd} / \mathrm{W}$ film) on hydrogen partial pressure at room temperature. The phases corresponding to $\alpha$-phase, plateau region and $\beta$-phase can be distinguished since the shape is similar to previously reported pressure-concentration isotherm.

decreased and the region of $\alpha$ - and $\beta$-phase separated clearly (Frazier and Glosser 1980; Lee and Glosser 1985).

\section{Conclusion}

The recovery behaviour of the optical hydrogen sensor using Pd thin film was investigated. It was found that the ehange in reflectance with hydrogen desorption indi- cated distinctive recovery characteristics with three-stage curve which depended on thickness of the Pd hydride film. Moreover, repetitional detection of hydrogen gas induced slower and more distinct three-stage desorption. The phase boundary of the Pd hydride was determined by the total reflectance change as a function of hydrogen partial pressure, and was correlated with the three-stage desorption. The dependencies of three-stage dehydrogenation on thickness of the Pd film and on the number of absorption-desorption cycles were related to the sharpening of pressure concentration isotherm.

\section{Acknowledgement}

This work was supported by a Grant-in-Aid for Science Research from the Ministry of Education, Science, Sports and Culture (No. 07555192).

\section{References}

Christofides C and Mandelis A $1990 \mathrm{~J}$. Appl. Phys. $68 \mathrm{R} 1$

Frazier G A and Glosser R 1980 J. Less-Common Metals 74 89

Hamagami J, Huybrechts B and Takata M 1999 Sensors and Actuators B (to be published)

Lee M and Glosser R 1985 J. AppL Phys. 575236

Lewis F A 1967 The palladium hydrogen system (New York: Academic Press) chap. 2 and 4

Oh Y, Hamagami J, Watanabe $Y$, Takata $M$ and Yanagida $H$ 1993 J. Ceram. Soc. Jpn 101618

Okuhara Y, Imai Y, Noguchi Y and Takata M 1999 Sensors and Actuators $\mathrm{B}$ (to be published) 